\title{
Raman and Infra-red properties and layer dependence of the phonon dispersions in multi-layered graphene
}

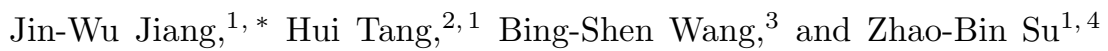 \\ ${ }^{1}$ Institute of Theoretical Physics, Chinese Academy of Sciences, Beijing 100080, China \\ ${ }^{2}$ Institute of Physics, Chinese Academy of Sciences, Beijing 100190, China \\ ${ }^{3}$ State Key Laboratory of Semiconductor Superlattice and Microstructure \\ and Institute of Semiconductor, Chinese Academy of Sciences, Beijing 100083, China \\ ${ }^{4}$ Center for Advanced Study, Tsinghua University, Beijing 100084, China
}

(Dated: October 27, 2018)

\begin{abstract}
The symmetry group analysis is applied to classify the phonon modes of $N$-stacked graphene layers (NSGL's) with AB- and AA-stacking, particularly their infra-red and Raman properties. The dispersions of various phonon modes are calculated in a multi-layer vibrational model, which is generalized from the lattice vibrational potentials of graphene to including the inter-layer interactions in NSGL's. The experimentally reported red shift phenomena in the layer number dependence of the intra-layer optical C-C stretching mode frequencies are interpreted. An interesting low frequency inter-layer optical mode is revealed to be Raman or Infra-red active in even or odd NSGL's respectively. Its frequency shift is sensitive to the layer number and saturated at about 10 layers.
\end{abstract}

PACS numbers: 81.05.Uw, 63.22.+m

\section{INTRODUCTION}

In recent years, the ultrathin graphite films, i.e. $N$ (>1) stacked graphene layers (NSGL's), are successfully fabricated $\stackrel{1,2}{i}$ Extensive studies have been devoted to these systems due to their competitive capability for the design of novel nano-devices. It's desirable to carry out a through symmetry analysis to figure out the corresponding electron and phonon spectra, and to reveal the relevant selection rules and the optical activities. Moreover, to our knowledge, although there are some studies on the electronic structure,,$\underline{\underline{3}}$ no existing works concern the phonon dispersions for the NSGL's. Recently it is reported that the frequencies of optical C-C stretching mode in the NSGL's decrease with increasing $N \underline{\underline{4}, 5, \underline{6}}$ The amplitude of this red shift is about $3-5,5-6$, and $8 \mathrm{~cm}^{-1}$ in these three experiments respectively. Theoretical explanation of this red shift is required.

In this paper, the symmetry analysis is referred for both the AB- and AA-stacked lattice structures, while the latter has the point group $D_{6 h}$ irrespective of the even-oddness of $N$. We classify the phonon normal modes at $\Gamma$ point and determine their Raman and Infrared (Ir) properties. We generalize the force constant model of graphene ${ }^{7}$ into NSGL's and it can be applied to calculate the phonon dispersions for the NSGL's in ABor AA-stacking with arbitrary layer number $N$.

For the intra-layer optical C-C stretching mode with frequency around $1600 \mathrm{~cm}^{-1}$, it is Raman active for all NSGL's and the calculated frequencies exhibit layernumber dependence as that the frequency decreases with $N$ increasing. The red shift values for the AB- (AA-) stacked systems are about $2 \mathrm{~cm}^{-1}\left(4 \mathrm{~cm}^{-1}\right)$ which are in consistent with the experimental measurements. In the medium frequency range around $800 \mathrm{~cm}^{-1}$, the out-ofplane optical mode is Ir active for AB-stacked structure but neither Raman nor Ir active in AA-stacking. Its fre- quency behaviors a blue shift as layer number increasing. There is an interesting inter-layer optical mode in the low frequency region which is Raman active in the NSGL's with $N$ even (ENSGL's) while Ir active in NSGL's with $N$ odd (OENSGL's). Its frequency value depends on the layer number $N$ more sensitively and increases from $106 \mathrm{~cm}^{-1}\left(94.5 \mathrm{~cm}^{-1}\right)$ to $149.8 \mathrm{~cm}^{-1}\left(133.6 \mathrm{~cm}^{-1}\right)$ for the AB- (AA-) stacked NSGL's, which is of an order less than those intra-layer optical modes. Phonon dispersions for the AA-stacked 3-dimensional (3D) graphite are also discussed.

The present paper is organized as follows. In Sec. II, the lattice configuration is illustrated for the NSGL's. Sec. III is devoted to the symmetry analysis for the phonon modes. The vibrational potential energy is discussed in Subsec. IV A, while the main results and relevant discussions on phonon spectrum calculations are presented in Subsec. IV B. The paper ends with a brief summary in Sec. V.

\section{LATTICE CONFIGURATION}

\section{A. AB-stacked}

It is known that graphene is a single layer of carbon atoms with the honeycomb lattice configuration which is characterized by the $D_{6 h}$ symmetry ${ }^{-8}$ The $3 \mathrm{D}$ graphite is AB-stacked honeycomb lattice, where the $\mathrm{B}$ layers are achieved by shifting the A layers along one of its firstnearest carbon-carbon bonds in the horizontal plane as shown in Fig. 1(a). The space group of the 3D graphite is non-symmorphic group $D_{6 h}^{4}$ with non-primitive translation $\vec{\tau}=\frac{1}{2} \vec{c}$ (primitive translation $\vec{t}=n_{1} \vec{a}_{1}+n_{2} \vec{a}_{2}+n_{3} \vec{c}$ ) $\frac{9}{\underline{9}}$ The distance between two adjacent layers is about $\frac{c}{2}=$ $3.35 \AA$ which is much larger than the bond length between 


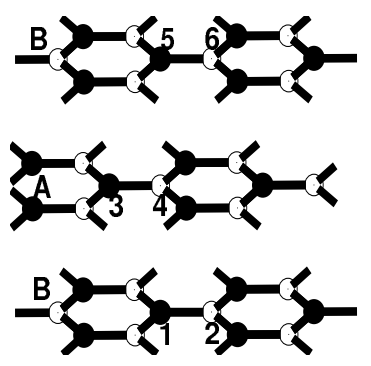

(a)

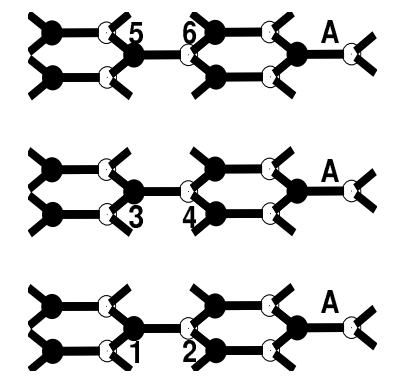

(b)
FIG. 1: The sketch of the configurations of AB-stacked in (a) and AA-stacked in (b) for multi-layer graphene.

two nearest-neighbor atoms in the plane, $b=1.42 \AA$.

The NGSL's are also constructed by AB-stacked honeycomb lattice, but with limited number of layers. Although the structures of each layer of 3D graphite and NSGL's are the same, the corresponding symmetry groups are different since the displacement symmetry along $\vec{c}$ axis no longer exists for NSGL's, so does the symmetry associated with $\vec{\tau}$. Now the symmetry group becomes a direct product of a $3 \mathrm{D}$ point group and a 2D translational group. And the point groups are different for ENSGL's and ONSGL's as mentioned in Ref. 10. For the ENSGL's, a center of inversion $\sigma_{i}$ occurs in the middle of atom 4 in the $\frac{N}{2}$-th layer and 5 in the $\frac{N}{2}+1$-th layer as shown in Fig. 1)(a). There are one 3 -fold main axis in the direction perpendicular to the layers and three 2-fold axes, $C_{2}^{\prime \prime}$, perpendicular to the main axis and at angles of $\pi / 3$ to each other. All these symmetry operations together constitute the point group $D_{3 d}=\left\{E, 3 C_{3}, 3 C_{2}^{\prime \prime}\right\} \times\left\{E, \sigma_{i}\right\}$. In the ONSGL's, instead of a center of inversion there is a reflection symmetry $\sigma_{h}$ with the middle layer as its reference plane. A 3 -fold main axis exists in the direction of $z$-axis and three 2-fold axes, $C_{2}^{\prime}$, are perpendicular to it. We notice that these three 2 -fold axes $C_{2}^{\prime}$ are one to one perpendicular to those of $C_{2}^{\prime \prime}$. Consequently, the symmetry group for the ONSGL's is $D_{3 h}=\left\{E, 3 C_{3}, 3 C_{2}^{\prime}\right\} \times\left\{E, \sigma_{h}\right\}$.

The environments for an atom in the graphite and NSGL's are different from that in 2D graphene. For each carbon atom in the graphene layer, there are three nearest-neighbor carbon atoms and six next-nearestneighbors. There are four carbon atoms 1, 2, 3, 4 in a unit cell of graphite, as represented in Fig.1(a). For atom 4 in A layer, there are two inter-layer nearest-neighbors in each of the two adjacent layers, with the distance $c$. Also, in each of the two adjacent layers, there are three inter-layer next-nearest-neighbor atoms around atom 4 with distance $\sqrt{b^{2}+\left(\frac{c}{2}\right)^{2}}$. As illustrated in Fig. 1(a), the adjacent environment for atom 3 is quite different from that of atom 4 . It has no inter-layer first-neighbors with the same distance as $\frac{c}{2}$. However, atom 3 has six interlayer neighbors in each of the two adjacent layers with the same distance as that of the second-nearest-neighbor of atom 4 .

\section{B. AA-stacked}

The AA-stacked NSGL's (AA-NSGL's) are constructed by AA-stacked honeycomb lattice where all layers have the same configuration. In the AA-stacked system, the ENSGL's, ONSGL's and the 3D graphite have the same point group $D_{6 h}$ which is the symmetry of the graphene. As shown in Fig. 1(b), the environments for carbon atoms in the AA-stacked NSGL's are quite different from those in AB-stacked systems. For each atom, there are two inter-layer nearest-neighbors in each of the two adjacent layers with the distence $\frac{c}{2}$. And each atom has six inter-layer second-nearest-neighbors with distance $\sqrt{b^{2}+\left(\frac{c}{2}\right)^{2}}$ in its two adjacent layers. We notice here that in the AA-stacked 3D graphite, there are only two atoms in the unit cell and the primitive translation along $c$-axis is $\vec{c} / 2$, which is half of the correspondence in the AB-stacked 3D graphite.

\section{SYMMETRY ANALYSIS FOR THE PHONON MODES}

The dynamical representation $\Gamma^{d y n}=\Gamma^{v} \otimes \Gamma^{\text {atom }}$ can be decomposed into the irreducible representations of the symmetry group, with the lattice displacements as the bases, where $\Gamma^{v}$ is the vector representation and $\Gamma^{\text {atom }}$ is the permutation representation of the group. By applying the projection operator technique, we carry out the decomposition of the dynamical representation into the irreducible representations for the NSGL's with $N$ even and odd respectively. According to Elliott, 11 the Ir active phonon modes belong to the irreducible representations decomposed from the vector representation $\Gamma^{v}$, while the Raman active modes correspond to the irreducible representations shown up in the decomposition of a six-dimensional representation with bases as the quadratic forms: $x^{2}+y^{2}, z^{2}, x^{2}-y^{2}, x y, y z$, and $z x$. The three acoustic modes with zero frequency at the $\Gamma$ point, which correspond to the vector representation $\Gamma^{v}$, are excluded in the consideration of Ir and Raman active modes. For comparison the corresponding results for the graphene and 3D graphite are also listed in the following. The symbol for the irreducible representations we used here is the notation used in Ref. 12 which is the most commonly used in the treatment of molecules.

The symmetry analysis for the phonon modes and the Raman and Ir modes are classified in Table. I In the ABstacked NSGL's, since the $\sigma_{i}$ symmetry and $\sigma_{h}$ symmetry can not coexist in the ENSGL's or ONSGL's, we can see two straightforward consequences from the above classification for the Ir and Raman active modes. Firstly, in the ENSGL's, phonon modes can not be Ir and Raman active simultaneously (which is also true for the gaphene and 
TABLE I: The symmetry analysis for the phonon modes at the $\Gamma$ point of the NSGL's with AA- or AB-stacking. Phonon modes are classified by the irreducible representations of $\Gamma^{d y n}$ in the fourth column. The irreducible representations of the Ir and Raman active modes are listed in the fifth and sixth column respectively.

\begin{tabular}{|c|c|c|c|c|c|}
\hline & & group & $\Gamma^{d y n}$ & $\Gamma^{I r}$ & $\Gamma^{R}$ \\
\hline graphene $^{8}$ & & $D_{6 h}$ & $A_{2 u} \oplus B_{2 g} \oplus E_{1 u} \oplus E_{2 g}$ & 1 & $E_{2 g}$ \\
\hline AB-stacked & $\begin{array}{c}\text { ENSGL's } \\
\text { ONSGL's } \\
3 \mathrm{D}^{13}\end{array}$ & $\begin{array}{l}D_{3 a^{\frac{10}{\underline{L}}}} \\
D_{3 h^{\frac{10}{}}} \\
\\
D_{6 h}^{4}\end{array}$ & $\begin{array}{c}N\left(A_{1 g} \bigoplus A_{2 u} \bigoplus E_{g} \bigoplus E_{u}\right) \\
(N-1) A_{1}^{\prime} \bigoplus(N+1) A_{2}^{\prime \prime} \bigoplus(N+1) E^{\prime} \\
\bigoplus(N-1) E^{\prime \prime} \\
2\left(A_{2 u} \bigoplus B_{2 g} \bigoplus E_{1 u} \bigoplus E_{2 g}\right)\end{array}$ & $\begin{array}{c}(N-1) A_{2 u} \bigoplus(N-1) E_{u} \\
N A_{2}^{\prime \prime} \bigoplus N E^{\prime} \\
A_{2 u} \bigoplus E_{1 u}\end{array}$ & $\begin{array}{c}N A_{1 g} \bigoplus N E_{g} \\
(N-1) A_{1}^{\prime} \bigoplus N E^{\prime} \bigoplus(N-1) E^{\prime} \\
2 E_{2 g}\end{array}$ \\
\hline AA-stacked & $\begin{array}{c}\text { ENSGL's } \\
\text { ONSGL's } \\
3 \mathrm{D}\end{array}$ & $\begin{array}{l}D_{6 h} \\
D_{6 h} \\
D_{6 h}\end{array}$ & $\begin{array}{c}\frac{N}{2}\left(A_{1 g} \bigoplus A_{2 u} \bigoplus B_{1 u} \bigoplus B_{2 g} \bigoplus E_{1 u}\right. \\
\left.\bigoplus E_{1 g} \bigoplus E_{2 g} \bigoplus E_{2 u}\right) \\
\frac{N-1}{2}\left(A_{1 g} \bigoplus B_{1 u} \bigoplus E_{1 g} \bigoplus E_{2 u}\right) \\
\bigoplus \frac{N+1}{2}\left(A_{2 u} \bigoplus B_{2 g} \bigoplus E_{1 u} \bigoplus E_{2 g}\right) \\
A_{2 u} \bigoplus B_{2 g} \bigoplus E_{1 u} \bigoplus E_{2 g}\end{array}$ & $\begin{array}{c}\left(\frac{N}{2}-1\right)\left(A_{2 u} \bigoplus E_{1 u}\right) \\
\frac{N-1}{2}\left(A_{2 u} \bigoplus E_{1 u}\right) \\
/\end{array}$ & $\begin{array}{c}\frac{N}{2}\left(A_{1 g} \bigoplus E_{1 g} \bigoplus E_{2 g}\right) \\
\frac{N-1}{2}\left(A_{1 g} \bigoplus E_{1 g}\right) \bigoplus \frac{N+1}{2} E_{2 g} \\
E_{2 g}\end{array}$ \\
\hline
\end{tabular}

graphite). However, in the ONSGL's, the $N E^{\prime}$ modes are both Ir and Raman active. This is because there is no inversion center in the ONSGL's. Secondly, among the optical modes with their vibrational displacements perpendicular to the constituent layers, there is an exotic mode oscillating with each layer as a whole but alternatively from layer to layer. It belongs to the $A_{1 g}$ in the ENSGL's and $A_{2}^{\prime \prime}$ in the ONSGL's. Since the $\sigma_{h}$ operation exists only in the ONSGL's, this mode ( $\omega_{1}$ mode) is Raman active in the ENSGL's while Ir active in the ONSGL's.

In the AA-stacked NSGL's with $N$ even or odd, the symmetry group is $D_{6 h}$ which includes both $\sigma_{i}$ and $\sigma_{h}$. As a result, phonon modes can not be Ir and Raman active simultaneously. The $\omega_{1}$ mode mentioned above belongs to the $A_{1 g}$ in the ENSGL's and $A_{2 u}$ in the ONSGL's. This mode is Raman active in the ENSGL's while Ir active in the ONSGL's which is the same as the ABstacked NSGL's. Nevertheless, its vibrational mode favors to take the maximum advantage of the inter-layer interactions. It would be sensitive and useful experimentally to identify the even-oddness of the NSGL's with a few layers.

\section{CALCULATION FOR THE PHONON DISPERSION}

\section{A. Vibrational potential energy}

The vibrational potential energy for a graphene sheet can be described by five quadratic terms with the rigid rotational symmetry implemented $, \underline{7}, \underline{14}$ They are the 1 st and 2nd nearest-neighbor stretching, the in-plane bond angle variations, the out-of-surface bond bending and the bond twisting energies. From the modality of atomic movements, we can also classify the inter-layer vibrational potential terms into three types: The first one describes the stretching movements between the two atoms located in
TABLE II: Comparison of several mode frequencies (in the unit of $\mathrm{cm}^{-1}$ ) for the AB-stacked 3D graphite between the our calculation results and the experimental values. 19,20

\begin{tabular}{cllll}
\hline \hline Reps & $A_{1}^{\prime}$ & $E_{2 g}$ & $A_{2 u}$ & $E_{2 g}$ \\
\hline experiments & $30^{19}$ & $40^{19}$ & $868^{20}$ & $1586^{20}$ \\
theory & 30.2 & 42.7 & 869.9 & 1586.6 \\
\hline \hline
\end{tabular}

the adjacent layers. The second describes the relative movement between the two pairs of atoms with a common one as an apex. That is this type of movements involves three atoms to form one bond in a layer and another connecting the two nearest layers. The third involves more than three atoms according to the specific bond configurations. As shown in Fig. 1 there is only one inter-layer nearest-neighbor carbon-carbon bond in each unit cell (the bond between atoms 1 and 4). So that just the twisting potential on the inter-layer bond is encountered here. The whole of these terms is actually a modified valence force field model to account some interactions for far away atoms in response to the bond charge effect in certain extent. Since the inter-layer bonds are much longer than that in the plane, all above three type interactions are one or two orders less than their counterparts in layer and they themselves have comparable contributions. In the following, the inter-layer terms are written in the AB-stacked system and they can be similarly generalized to the AA-stacked system.

(1). The inter-layer bond stretching energies $V_{l}^{(i n t)}\left(V_{s l}^{(i n t)}\right)$ have the form as:

$$
\sum_{i, j} \frac{\hat{k}_{l}}{2}\left[\left(\vec{u}_{i}-\vec{u}_{j}\right) \cdot \vec{e}_{i j}^{l}\right]^{2},
$$

where $\vec{u}_{i}\left(\vec{u}_{j}\right)$ is the displacement vector of the atom $i(j)$ and $\vec{e}_{i j}^{l}$ is the unit vector from atom $i$ to atom $j$. If the summation is taken over the nearest-neighbored interlayer pair of atoms, the corresponding force constant is 
denoted as $\hat{k}_{l}$ while for next nearest-neighbor inter-layer pairs we have the force constant as $\hat{k}_{s l}$.

(2). For the three atoms 1,4 and $i$, where $i$ is the inplane nearest neighbor of atom 1 (see Fig. 11), we found that under a specific configuration with atom $i$ rather than atom 1 as an apex, while the force being along the corresponding bond direction instead of perpendicular direction, a correlation term $\hat{k}_{r r}$ has the most and sensitive contribution to the layer dependence of the inta-layer CC stretching optical modes,

$$
\frac{\hat{k}_{r r}}{2} \sum_{i}\left[\left(\vec{u}_{1}-\vec{u}_{i}\right) \cdot \vec{e}_{i 1}^{l}-\left(\vec{u}_{4}-\vec{u}_{i}\right) \cdot \vec{e}_{i 4}^{l}\right]^{2} .
$$

Actually the two square terms in above modality have already been accounted in the in-plane and inter-plane stretching terms respectively. Only the across term is left,

$$
V_{r r}=-\hat{k}_{r r} \sum_{i}\left[\left(\vec{u}_{1}-\vec{u}_{i}\right) \cdot \vec{e}_{i 1}^{l}\right]\left[\left(\vec{u}_{4}-\vec{u}_{i}\right) \cdot \vec{e}_{i 4}^{l}\right]
$$

which weakens the interaction between two adjacent layers. The positive definite condition for getting real frequencies is $\hat{k}_{s l} \geq \hat{k}_{r r}$.

(3). The twisting potential for an inter-layer bond between atoms 1 and 4 is coming from the two sets of three nearest-neighbors of atoms 1 and 4 respectively. It can be described as

$$
V_{t w}=\frac{\hat{k}_{t w}}{2}\left[\sum_{i}\left(\vec{u}_{i}-\vec{u}_{1}\right) \cdot \vec{e}_{i}^{\theta}-\sum_{j}\left(\vec{u}_{j}-\vec{u}_{4}\right) \cdot \vec{e}_{j}^{\theta}\right]^{2}
$$

where $\sum_{i}$ and $\sum_{j}$ represent the summation over the three intra-plane first-nearest-neighbors for atoms 1 and 4 respectively. $\vec{e}_{i}^{\theta}=\vec{e}_{z} \times \vec{e}_{1 i}^{t}$ is the tangential unit vector in the plane formed by three atoms 1,4 , and $i$. The expression in quadratic form as a whole ensures a proper definition for the torsion angle. For pure rotations around the bond, this expression gives zero torsion consistently. In contrast, the bond is most severely twisted when the three neighbors around atom 1 and those of atom 4 rotate reversely.

We stress here that, all of the above four inter-layer vibrational potential energy terms satisfy the rigid rotational symmetry requirements $\frac{14,15,16}{}$ which guarantees the existence of the flexure modes in the low dimensional systems. Although we establish the vibrational potential terms based on the analysis to the modality of movements, the bond charge effect especially along the perpendicular direction has been involved by extending the valence force field beyond the nearest neighbors. Comparing, for example, the above $\hat{k}_{r r}$ term with that $V_{b-b}$ in Ref. 17, which is followed from the bond-charge model, they have the same negative cross term.

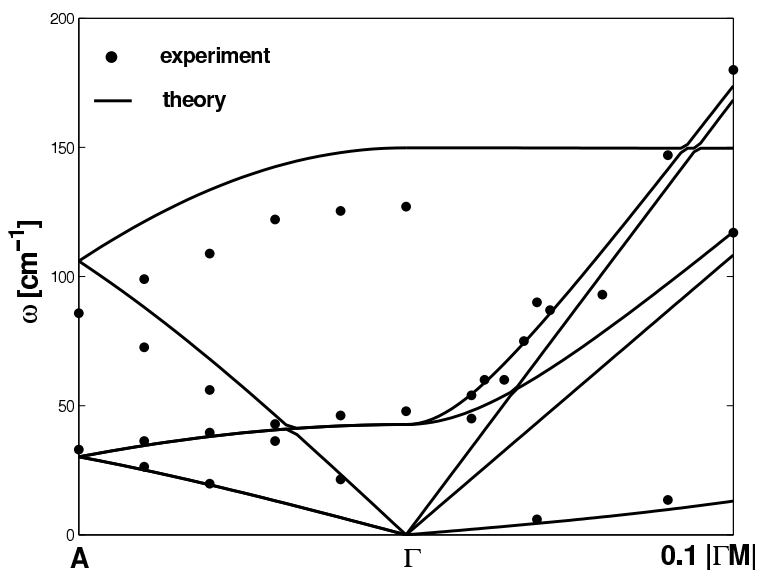

FIG. 2: Phonon dispersion for the 3D graphite in the lowfrequency region. Solid dots are the experimental results of Ref. 19. Our theoretical calculations are shown in lines.

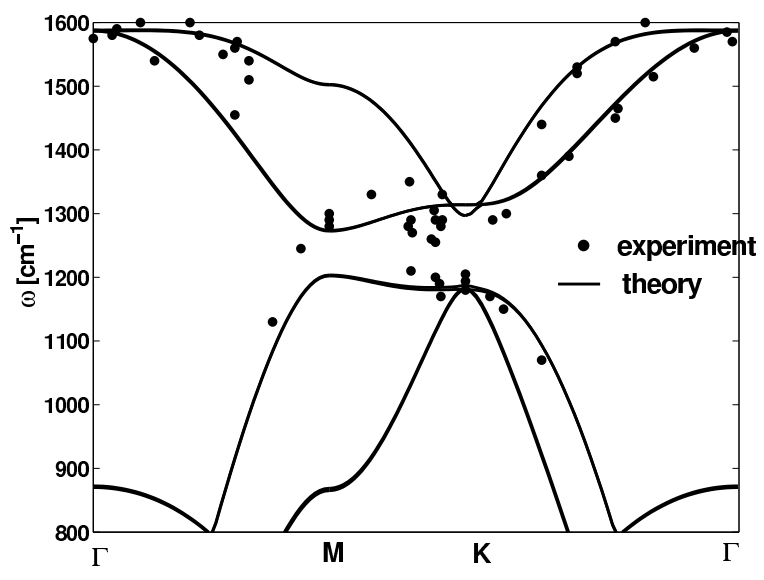

FIG. 3: Phonon dispersion for the 3D graphite in the highfrequency region. Solid dots are the experimental results..$^{20,21}$ In Refs. 20 21, those phonon wave vectors $\vec{q}$, which were not exactly along the $\Gamma-\mathrm{M}$ or $\Gamma-\mathrm{K}-\mathrm{M}$ direction, were projected onto the closest high-symmetry direction. Lines are our theoretical calculations.

\section{B. Results and discussion}

The five intra-layer force constants we used in the following are taken from Ref. 18 with a minor modification. We adjust the four inter-layer force constants to fit the experimental values of four modes in $3 \mathrm{D}$ graphite as shown in Table II] The fitting error for phonon modes is kept less than $7 \%$. The inter-layer force constants are then fitted as $\hat{k}_{l}=0.77 \mathrm{Nm}^{-1}, \hat{k}_{s l}=0.95 \mathrm{Nm}^{-1}$, $\hat{k}_{t w}=0.64 \mathrm{Nm}^{-1}, \hat{k}_{r r}=0.9 \mathrm{Nm}^{-1}$.

Base on the above fitted vibrational potential energy with nine terms, we calculate the dispersion curves for the AB-stacked graphite. As illustrated in Figs 2 and 3 , our theoretical calculations meet the experimental results not only in the low frequency $\frac{19}{9}$ but also in the high fre- 
TABLE III: The Raman and Ir mode frequencies (in the unit of $\mathrm{cm}^{-1}$ ) for the AB-stacked 3D graphite, AB-stacked 2-layer and AA-stacked 3D grphite are listed. The irreducible representations are presented in the brackets following the frequency values.

\begin{tabular}{|l|cc|ll|}
\hline \hline & Raman & Infra-red \\
\hline AB- 3D & $42.7\left(E_{2 g}\right)$ & $1586.7\left(E_{2 g}\right)$ & $869.9\left(A_{2 u}\right)$ & $1588.2\left(E_{1 u}\right)$ \\
\hline AB- & $30.2\left(E_{g}\right)$ & $106\left(A_{1 g}\right)$ & $868.7\left(A_{2 u}\right)$ & $1588.1\left(E_{u}\right)$ \\
2-layer & $867.4\left(A_{1 g}\right)$ & $1587.3\left(E_{g}\right)$ & & \\
\hline AA- 3D & $1584.7\left(E_{2 g}\right)$ & & \\
\hline \hline
\end{tabular}

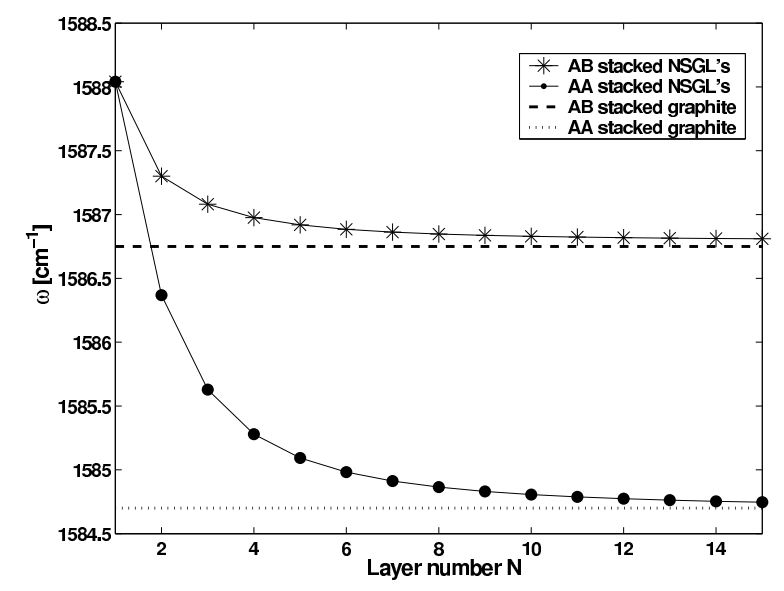

FIG. 4: The frequency value for the optical C-C stretching mode vs the layer number $N$. Lines are draw to guide eyes.

quency regions..$^{20,21}$ The excellent consistency with the experimental data shows that our model and parameters are reasonable and applicable.

With the above force constants, we can calculate the phonon dispersion for NSGL's of the AA- or AB-stacking with an arbitrary layer number $N$. In Fig. 4, the calculated frequency of the intra-layer optical C-C stretching mode is represented with different stacked styles and layer number $N$. The layer dependence of the frequency shows up a red shift behavior which is in agreement with the experimental measurements. The frequency value for this mode is about $1588 \mathrm{~cm}^{-1}$ in the single graphene layer and decreases with increasing $N$ and almost saturates at $N=10$. The limit is $1586.7 \mathrm{~cm}^{-1}\left(1584.7 \mathrm{~cm}^{-1}\right)$ in the AB- (AA-) stacked system respectively. The amount of red shift value in our calculation corresponds excellently with that measured by experiments within the ranges $3-5,5-6$, and $8 \mathrm{~cm}^{-1}$ in Refs. 4,5 and 6 , respectively.

The out-of-plane optical mode, belonging to the $A_{2 u}$ $\left(B_{2 g}\right)$ irreducible representation in the AB (AA) stacking, is Ir active in the AB-stacking yet inactive in the AA-stacking irrespective of the even-oddness of the layer number $N$ and is useful in determining whether the NSGL's is of AB or AA stacking. As shown in Fig. 5 , frequencies for this mode depend on the layer number $N$

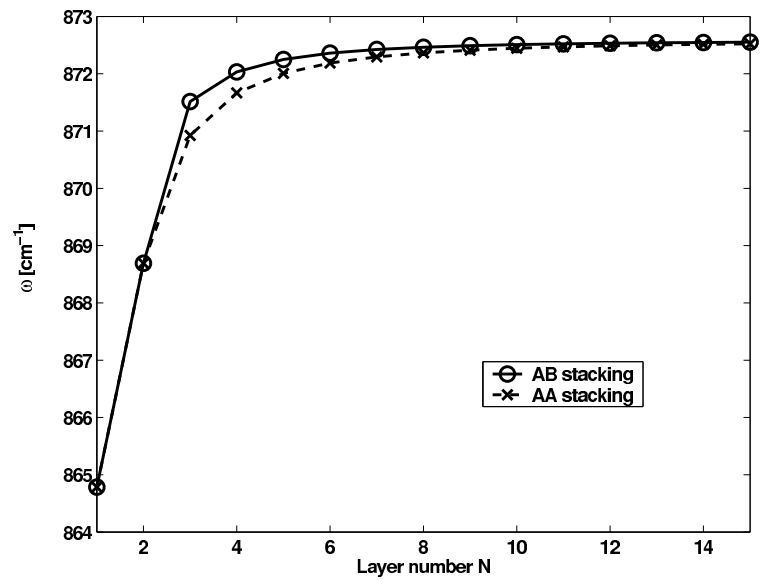

FIG. 5: The frequency value for the out-of-plane optical mode vs the layer number $N$. This mode is Ir active in the AB stacking while it is neither Ir nor Raman active in the AA stacking. Lines are draw to guide eyes.

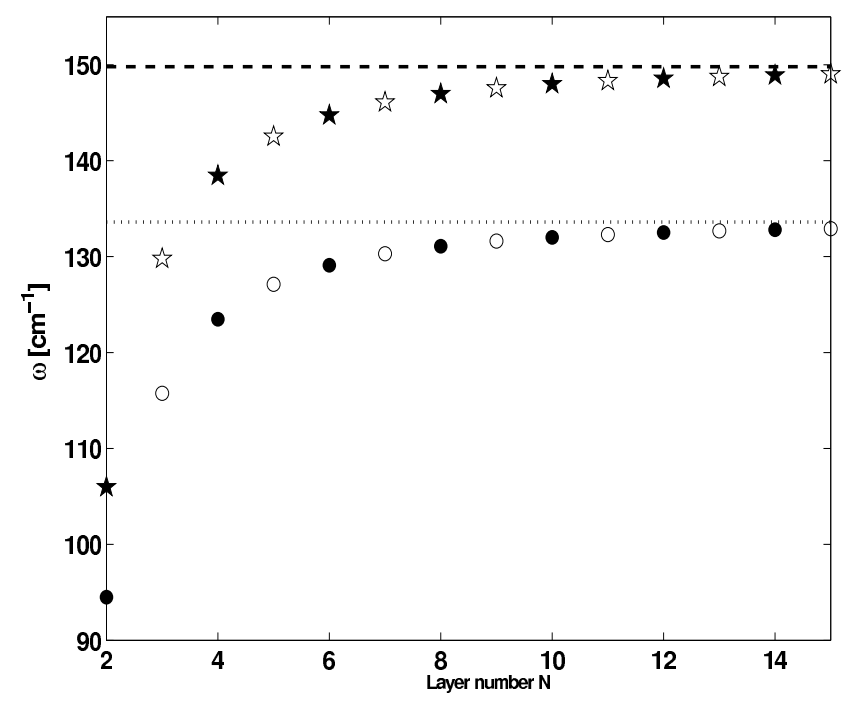

FIG. 6: The frequencies of the inter-layer optical mode vs the layer number $N$. Datas for the AB and AA stacked NSGL's are designated by pentagrams and circles, respectively. The Raman and Infra-red activities for this mode are displayed by the full and empty symbols, respectively. The broken and dashed lines correspond to the frequencies for the AB-stacked and AA-stacked graphite, respectively.

and increase from $864.8 \mathrm{~cm}^{-1}$ to $872.6 \mathrm{~cm}^{-1}$ in both the $\mathrm{AB}$ and $\mathrm{AA}$ stacking. In contrast to the $\mathrm{C}-\mathrm{C}$ stretching optical mode, this mode frequency exhibits a blue shift type layer dependence which could be identified with the development of the experimental technique.

For the inter-layer optical mode, the layer number dependence of the frequency value is shown in Fig. 6. This mode takes the greatest advantage of the inter-layer interation and is considerably dependent on the layer number $N$ and the stack style $\mathrm{AB}$ or $\mathrm{AA}$. In case of $N=2$, 


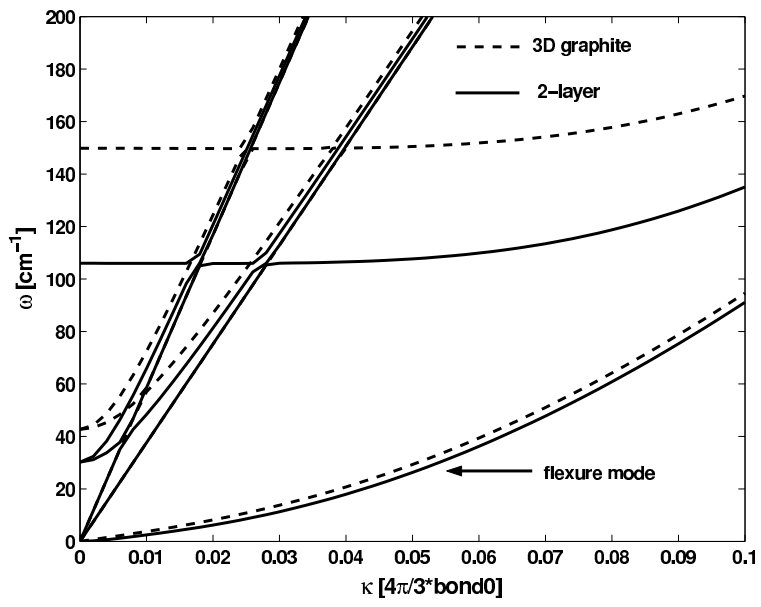

FIG. 7: In the low frequency region, there is significant difference between 3D graphite and the 2-layer graphene.

the $\omega_{1}$ mode has the frequency values $106 \mathrm{~cm}^{-1}$ and $94.5 \mathrm{~cm}^{-1}$ for the AB- and AA-stacked NSGL's respectively. The frequencies of the $\omega_{1}$ mode increase with increasing $N$ and almost come to the limit values at $N=10$. The limit values are $149.8 \mathrm{~cm}^{-1}$ and $133.6 \mathrm{~cm}^{-1}$ for the AB- and AA-stacked NSGL's respectively. The frequency differences as well as the Raman versus Ir (see Sec. III) of the $\omega_{1}$ mode in NSGL's with different layers might inspire considerably experimental interest in the $\omega_{1}$ mode.

We then calculate the phonon dispersion for the 2layered $\mathrm{AB}$ stacking, in comparison with that of the $3 \mathrm{D}$ graphite. The most significant difference between the 2-layer graphene and the 3D graphite lies in the lowfrequency region around the $\Gamma$ point as shown in Fig. 7 . The frequencies of the low-frequency optical modes in the 2-layer graphene are much smaller than their counterparts in the 3D graphite. The frequencies of the Raman and Ir active modes are shown in the third line of Table III among which the two $A_{1 g}$ modes have the frequency value $\omega_{1}=106 \mathrm{~cm}^{-1}$ and $\omega_{2}=867.4 \mathrm{~cm}^{-1}$. In fact, $\omega_{1}$ and $\omega_{2}$ modes are the above mentioned $N A_{1 g}$ modes of the ENSGL's specified to $N=2$.

We further calculate the phonon dispersion curve for the AA-stacked 3D graphite as shown in Fig. 8. Since the unit cell contains only two atoms in contrast to that of the AB-stacked graphite, there are six branches of phonon dispersion. Along $\Gamma A$ in the Brillouin zone, the lowest and highest branches which correspond to the in-plane acoustic and optical vibrational modes are doubly degenerate, while the remaining two branches describing the out-of-plane vibration are non-degenerate. At the $A$ point in the Brillouin zone, there is a phase factor differ- ence of $\pi$ between two adjacent layers for the out-of-plane motion, among which the $\omega_{1}$ mode has the frequency value of $133.6 \mathrm{~cm}^{-1}$. In the fourth line of Table III. the Raman and Ir active modes for the AA-stacked 3D graphite are listed which are to be confirmed in future

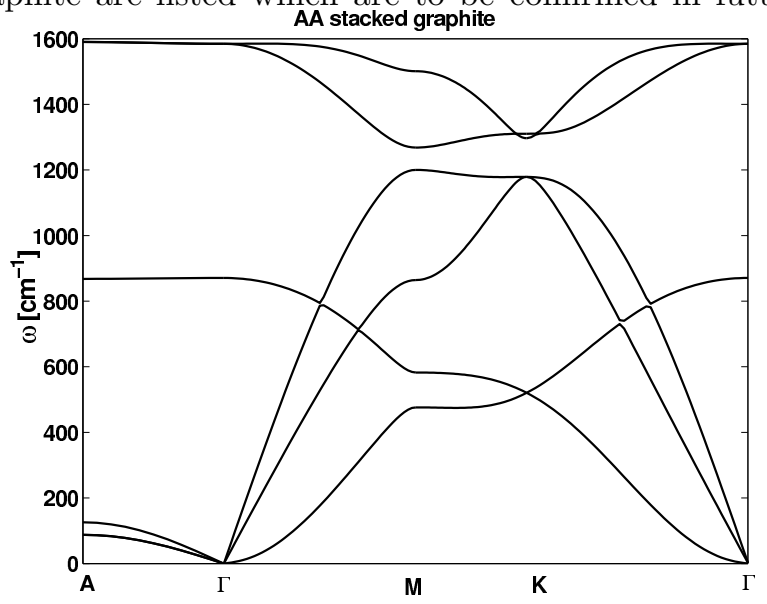

FIG. 8: The phonon dispersion along some high-symmetry directions for the AA-stacked 3D graphite. There are only six branches in the figure, since the unit cell in the AA-stacked $3 \mathrm{D}$ graphite contains two atoms.

experiments.

\section{CONCLUSION}

Based upon a thorough investigation of the lattice symmetry of the NSGL's, the Raman and Ir properties, in particular its layer dependence, is systematically studied. With a proposed generalized vibrational potential, we further calculate the phonon dispersion of various modes of the AB- or AA-stacked NSGL's where the layer dependence is also stressed. The calculated frequencies of optical C-C stretching mode exhibit a red shift as layer number increasing in both the AB- and AA-stacked NSGL's, and the shift value $2 \mathrm{~cm}^{-1}\left(4 \mathrm{~cm}^{-1}\right)$ for $\mathrm{AB}$ - (AA-) stacked NSGL's is in good consistent with the experimental measurements. The out-of-plane optical mode with frequency around about $800 \mathrm{~cm}^{-1}$ is Ir active in AB-stacked structure yet neither Raman nor Ir active in AA-stacking. Its frequency shows a blue shift layer dependence. We also predict that the frequency of the interlayer optical mode increases with $N$ increasing. Since this mode is more sensitive to the layer number $N$, it should be experimentally interesting in determining the lattice structure properties of the NSGL's. 
Science 306, 666 (2004).

2 C. Berger, Z. Song, and T. Li et al., J. Phys. Chem. B 108, 19912 (2004).

3 B. Partoens and F. M. Peeters, Phys. Rev. B 74, 075404 (2006); Phys. Rev. B 75, 193402 (2007).

4 A. C. Ferrari, J. C. Meyer, and V. Scardaci et al., Phys. Rev. Lett. 97, 187401 (2006).

5 A. Gupta, Gugang Chen, and P. Joshi et al., Nano Lett., 6, 2667, (2006).

6 Anindya Das, Biswanath Chakraborty, and A. K. Sood, arXiv:cond-mat/0710.4610v1.

7 T. Aizawa, R. Souda, S. Otani, Y. Ishizawa, and C. Oshima, Phys. Rev. B 42, 11469 (1990); 43, 12060(E) (1991).

8 R. Saito, G. Dresselhaus, and M.S. Dresselhaus, Physical Properties of Carbon Nanotubes (Imperial College Press, 1998).

9 L. Brillson, E. Burskin, A. A. Maradudin, and T. Stark, The Physics of Semimetals and Narrow-Gap Semiconductors, Ed. D. L. Carter and R. T. Bate, (Pergamon Press, London/Oxford 1971).

10 J. L. Manes, F. Guinea, and Maria A. H. Vozmediano, Phys. Rev. B 75, 155424 (2007).

11 J. P. Elliott and P. G. Dawber, Symmetry in Physics, Vol.
1, (Macmillan Press, Ltd., 1979).

12 Eyring, Walter, and Kimball, Quantum Chemistry, John Wiley and Sons, (Inc., New York, 1940).

13 K. K. Mani and R. Ramani, Phys. Status Solidi B 61, 659 (1974).

14 J. W. Jiang, H. Tang, B. S. Wang, and Z. B. Su, Phys. Rev. B 73, 235434 (2006).

15 V.N. Popov, V.E. Van Doren, and M. Balkanski, Phys. Rev. B 61,3078( 2000).

16 G.D. Mahan, and Gun Sang Jeon, Phys. Rev. B 70, 075405 (2004).

17 Gun Sang Jeon, and G.D. Mahan, Phys. Rev. B 72, 155415 (2005).

18 Jin-Wu Jiang, Hui Tang, Bing-Shen Wang and Zhao-Bin $\mathrm{Su}$, J. Phys.: Condens. Matter 20, 045228 (2008) .

19 R. Nicklow, N. Wakabayashi, and H. G. Smith, Phys. Rev. B 5, 4951 (1972).

20 J. Maultzsch, S. Reich, and C. Thomsen et al., Phys. Rev. Lett. 92, 075501 (2004).

21 M. Mohr, J. Maultzsch, and S. Reich et al., Phys. Rev. B 76, 035439 (2007). 